\title{
Network-based bioinformatics analyses on molecular pathways and pharmacological properties of oleuropein
}

\author{
Sevgi Gezici ${ }^{\star}$ and Nazim Sekeroglu* \\ Gaziantep University, Faculty of Medicine, Department of Medical Biology and Genetics, Gaziantep, Turkey \\ * Kilis 7 Aralik University, Faculty of Agriculture, Department of Horticulture, Kilis, Turkey
}

\section{Article Info}

Article history

Received 12 October 2021

Revised 28 November 2021

Accepted 29 November 2021

Published Online 30 December 2021

\section{Keywords}

Bioinformatics

Molecular pathway

Network pharmacology

Oleuropein

Protein-protein interactions

\begin{abstract}
Oleuropein, a valuable nutraceutical with powerful antioxidant, anticancer, anti-inflammatory, and cardioprotective properties, is the most abundant polyphenolic compound in olive leaf, fruits, and olive oil from the olive tree (Olea europaea L., Fam. Oleaceae). Biological activities and nutritional value of oleuropein have been previously identified; however, the molecular mechanisms of its action and pharmacological properties based on bioinformatics analyses have not been systematically revealed yet. In this work, network-based bioinformatics approached were implemented to evaluate targets of oleuropein in human genomes and proteomes, interacting genes of oleuropein and probable modulated pathways. The oleuropein was input into the ChEBI database, and the targets of its were predicted using DIGEP-Pred, and then, top interacting genes were identified by gene cards database. Afterward, STRING and KEGG enrichment database were used to construct a protein-protein interaction (PPI) network and molecular targeting pathway network, respectively. A total of 21 genes coding proteins, i.e., APP, BCHE, CAT, CCND1, CDKN1A, CREB1, CYP3A4, EPHX2, FASN, GPX1, MAPK1, MAPK14, MMP7, MMP9, NFKB1, NGF, OXA1L, PTGS2, PTGS2, SOD1, and VCAM1 affected by oleuropein were identified by gene set enrichment analysis. Further, multiple pathways including TNF signaling pathway, Kaposi sarcoma-associated herpesvirus infection, human cytomegalovirus infection, prostate cancer pathway, microRNAs in cancer, endocrine resistance, proteoglycans in cancer, and IL-17 signaling pathway were also determined to be regulated by oleuropein. Results indicated that oleuropein exhibits highly active pharmacological activity as a cholesterol antagonist, anti-inflammatory agent and hepatoprotective. This study provides the network-based scientific researches that will most likely be useful in screening biological, molecular and pharmacological properties of oleuropein for clinical application in the human diseases.
\end{abstract}

\section{Introduction}

Oleuropein is a secoiridoid type of phenolic compound that consists of three structural subunits, including a polyphenol (hydroxytyrosol), asecoiridoid (elenolic acid), and a glucose molecule. It abundantly presents in olive (Olea europaea Linn.), which commonly known as zaytoon and an essential component of Mediterranean diets. The content of oleuropein in olives varies according to the developmental stages and is divided in to three stages; first is growth phase in which accumulation of oleuropein occurs; second stage is green ripening stage in which a decrease in the oleuropein content occurs; and third is the black ripening stage where the oleuropein level is very low. In other words, the concentration of oleuropein decreases in many olive cultivars depending on the maturation of olives; however, it increases in the leaves of the olive trees during the same period, which may probably occur to protect against herbivores (Ahamad et al., 2019; Sekeroglu et al., 2020; Aggarwal et al., 2021).

Corresponding author: Dr. Sevgi Gezici

Associate Professor, Gaziantep University, Faculty of Medicine Department of Medical Biology and Genetics, Gaziantep, Turkey E-mail: drsevgigezici@gmail.com; sevgigezici@gantep.edu.tr Tel.: +90-5376348061

Copyright (c) 2021 Ukaaz Publications. All rights reserved.

Email: ukaaz@yahoo.com; Website: www.ukaazpublications.com
Oleuropein, as well as olive oil, fruits and olive leaves, have gained a great interest in phytochemical and pharmacologic research, since oleuropein exerts beneficial biological effects such as antidiabetic, antiobesity, antioxidant, anticancer, antiatherogenic, hypolipidemic, antihypertensive, anti-inflammatory, cardioprotective, neuroprotective and hepatoprotective (Menendez et al., 2008; Notarnicola et al., 2011; Priore et al., 2014; Hadrich et al., 2016; Shi et al., 2017; Das and Gezici, 2018; Sherif and Al-Gayyar, 2018; Zhang et al., 2019).Thanks to wide range of biological and pharmacological properties of oleuropein, it has been used in traditional medicine from ancient times. It has also proven to reduce the risk of many diseases and conditions such as, oxidative stress, obesity, diabetes, cholesterol, cancer, hypertension, hepatitis B, cerebral hemorrhage, myocarditis, Alzheimer's, Parkinson, hemorrhagic, cystitis, hypothyroidism, and amyloid diseases (Corona et al., 2007; Notarnicola, 2011; De Nicoló et al., 2013; Carito et al., 2015; Feng et al., 2017; Castejan et al., 2019; Zhang et al., 2019; Gao et al., 2020; Hsu et al., 2021).

Although, studies have been conducted to reveal the biological activity of oleuropein, network-based molecular and pharmacological activities of oleuropein have not been proposed yet. Hence, we aimed to elucidate the probable interactions of oleuropein by geneset enrichment and network pharmacology analyses to provide a new approach to uncover the therapeutic mechanisms of oleuropein 
that will facilitate its future clinical applications in the treatment of diseases.

\section{Materials and Methods}

\subsection{Chemical properties and targets}

Chemical Entities of Biological Interest (ChEBI) database, a part of ELIXIR Core Data Resources, was used for dictionary of molecular entities and chemical properties of oleuropein (Hastings et al., 2016). The targets of oleuropein were identified using DIGEP-Pred (Prediction of drug-induced changes of gene expression profile) based on structural formula of oleuropein (Lagunin et al., 2013).

\subsection{Gene set enrichment analysis}

Gene cards, the human gene database, was used to determine probable interacting genes of oleuropein. Based on this database, top interacting genes were analyzed using unique gene cards identifiers (GC ids) and gene cards inferred cunctionality scores (GIFtS), provided by the gene loc Algorithm (Harel et al., 2009; Fishilevich et al., 2016).

\subsection{Protein-protein interaction (PPI) analysis}

STRING database was used to annotate the role of probable interacting genes and proteins associated with oleuropein. PPI network mapping was conducted on oleuropein and protein targets using the retrieval of interacting genes database with the species limited to "homo sapiens" and a confidence score > 0.4 (Wu et al., 2009; Athanasios et al., 2017).

\subsection{KEGG pathway analysis}

KEGG (kyoto encyclopedia of genes and genomes) is an integrated database of genes and genomes used for mapping pathways at molecular level. KEGG enrichment analysis was performed for construction the network regulated by oleuropein (Aoki-Kinoshita and Kanehisa, 2007; Kanehisa et al., 2017).

\section{Results}

\subsection{Chemical composition and molecular properties of} oleuropein

Oleuropein $\left(\mathrm{C}_{25} \mathrm{H}_{32} \mathrm{O}_{13}\right)$ is a glycosylated secoiridoid that the most valuable phenolic compound found in the olive tree, Olea europaea L. This molecule is an ester of hydroxytyrosol and glucoside of elenolic acid with a molecular mass of $540.518 \mathrm{~g} / \mathrm{mol}$. The chemical structure and molecular identification of oleuropein are presented in the Table 1.

Table 1: Chemical structure and molecular properties of oleuropein

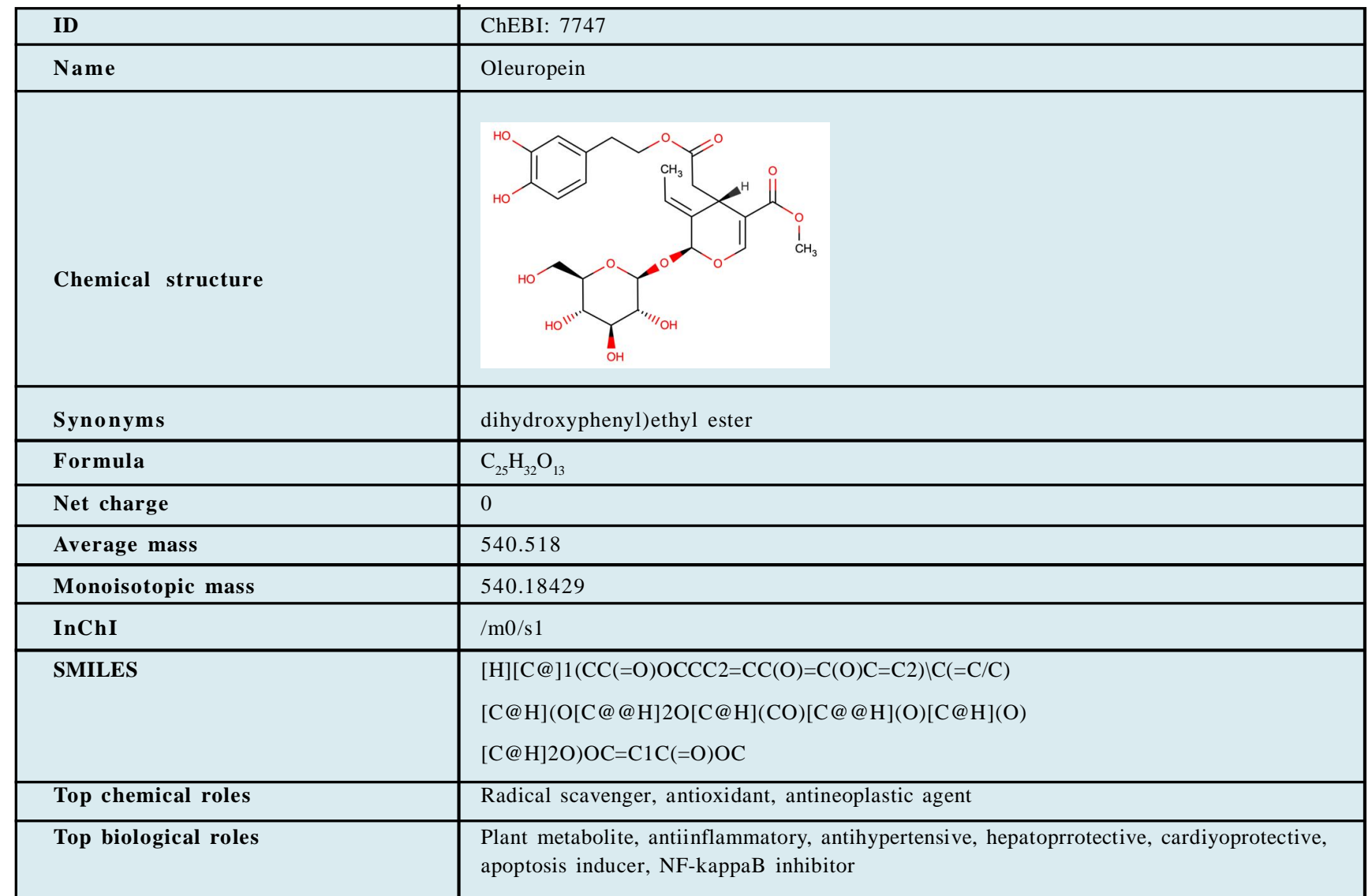

The targets of oleuropein were analyzed based on prediction of drug-induced changes of gene expression profile for proteins at the pharmacological activity $(\mathrm{Pa})>0.7$. The findings were summarized in the Table $2 . \mathrm{Pa}$ (probability to be active) means the chance that oleuropein is belonging to the subclass of active compounds, whilst
$\mathrm{Pi}$ (probability to be inactive) means the chance that oleuropein is belonging to the subclass of inactive compounds. According to the data presented in the table, oleuropein shows highly active pharmacological activity as a cholesterol antagonist, antiinflammatory agent and hepatoprotectant. 
Table 2: Prediction of drug-induced changes of gene expression profile for oleuropein

\begin{tabular}{|c|c|l|}
\hline $\mathbf{P a}$ & $\mathbf{P i}$ & Activity \\
\hline 0,706 & 0,006 & Chemopreventive \\
0,719 & 0,005 & Lactase inhibitor \\
0,726 & 0,020 & Anaphylatoxin receptor antagonist \\
0,739 & 0,006 & Anti-thrombotic \\
0,754 & 0,006 & Antihypercholesterolemic \\
0,788 & 0,005 & Vasodilator \\
0,802 & 0,017 & CYP2H substrate \\
0,810 & 0,016 & Benzoate-CoA ligase inhibitor \\
0,832 & 0,004 & Hepatoprotectant \\
0,869 & 0,016 & CDP-glycerol glycerophosphotransferase inhibitor \\
0,899 & 0,004 & Anti-inflammatory \\
0,933 & 0,002 & Cholesterol antagonist \\
\hline
\end{tabular}

Table 3: Top interacting genes with oleuropein

\begin{tabular}{|c|l|l|l|c|c|c|}
\hline SI. No. & Sy mbol & Description & Category & GIFtS & GCid & Score \\
\hline 1 & APP & Amyloid beta precursor protein & Protein Coding & 50 & GC21M025880 & 1.28 \\
2 & BCHE & Butyrylcholinesterase & Protein Coding & 48 & GC03M165772 & 0.21 \\
3 & CAT & Catalase & Protein Coding & 48 & GC11P034460 & 1.13 \\
4 & CCND1 & Cyclin D1 & Protein Coding & 51 & GC11P069641 & 1.44 \\
5 & CDKN1A & Cyclin dependent kinase inhibitor 1A & Protein Coding & 48 & GC06P061315 & 1.44 \\
6 & CREB1 & CAMP responsive element binding protein 1 & Protein Coding & 48 & GC02P207529 & 0.21 \\
7 & CYP3A4 & Cytochrome P450 family 3 subfamily A member 4 & Protein Coding & 50 & GC07M099759 & 0.21 \\
8 & EPHX2 & Epoxide hydrolase 2 & Protein Coding & 47 & GC08P027490 & 0.21 \\
9 & FASN & Fatty acid synthase & Protein Coding & 48 & GC17M082078 & 0.30 \\
10 & GPX1 & Glutathione peroxidase 1 & Protein Coding & 46 & GC03M049603 & 1.13 \\
11 & MAPK1 & Mitogen-activated protein kinase 1 & Protein Coding & 51 & GC22M021759 & 1.59 \\
12 & MAPK14 & Mitogen-activated protein kinase 14 & Protein Coding & 50 & GC06P061307 & 0.21 \\
13 & MMP7 & Matrix metallopeptidase 7 & Protein Coding & 48 & GC11M102425 & 1.52 \\
14 & MMP9 & Matrix metallopeptidase 9 & Protein Coding & 52 & GC20P046008 & 0.21 \\
15 & NFKB1 & Nuclear factor kappa B subunit 1 & Protein Coding & 51 & GC04P102501 & 1.44 \\
16 & NGF & Nerve growth factor & Protein Coding & 49 & GC01M115285 & 1.52 \\
17 & OXA1L & OXA1L mitochondrial inner membrane protein & Protein Coding & 38 & GC14P022766 & 0.21 \\
18 & PTGS1 & Prostaglandin-endoperoxide synthase 1 & Protein Coding & 45 & GC09P122370 & 1.13 \\
19 & PTGS2 & Prostaglandin G/H synthase 2 & Protein Coding & 48 & GC01M186640 \\
20 & SOD1 & Superoxide dismutase 1 & Protein Coding & 50 & GC21P031659 & 1.13 \\
21 & VCAM1 & Vascular cell adhesion molecule 1 & Protein Coding & 44 & GC01P100719 & 0.37 \\
\hline
\end{tabular}

\subsection{Top gene enrichment results}

A total of twenty-one genes were identified as top interacting genes that are regulated by oleuropein in human. All the genes are protein coding genes and NGF, APP, CYP3A4, MAPK1, CCND1, NFKB1, SOD1, MAPK14, and MMP7 were found the most interacting genes, whereas OXAIL, VCAM1, PTGS, and GPX1 were defined as the least interacting genes with oleuropein. The list of top genes interacts with oleuropein was given in the Table 3 .

\subsection{Construction of protein-protein interaction (PPI) network}

The relationship of a total of 21 proteins between each other were constructed from STRING database with FDR $<0.05$ and $\mathrm{p}$-value $=$ 1.33e-13. According to the interaction network diagram, MAPK14, CREB1, NFKB1, NGF, MMP9, APP, and PTGS2 are located in the center of the network (Figure 1).

\subsection{KEGG enrichment pathway analysis}

The probably modulated pathways were determined regarding the KEGG pathway database. According to the KEGG analysis, 116 different pathways were identified corresponding to 21 protein targets. The pathways modulated by oleuropein were presented in the Table 4. As can be seen in the Table 4, several target proteins are simultaneously involved in one pathway, while one target protein is also present in many pathways. TNF signaling pathway, Kaposi sarcoma-associated herpesvirus infection, human cytomegalovirus 
infection, hepatitis B, prostate cancer pathway, micro RNAs in cancer, human papillomavirus infection, endocrine resistance, proteoglycans in cancer, chemical carcinogens, pathways in neurodegeneration, hepatitis B, IL-17 signaling pathway and so forth were the top oleuropein-regulated pathways with the lowest false discovery rate $($ FDR $<0.05)$, and screened in Figure 2.

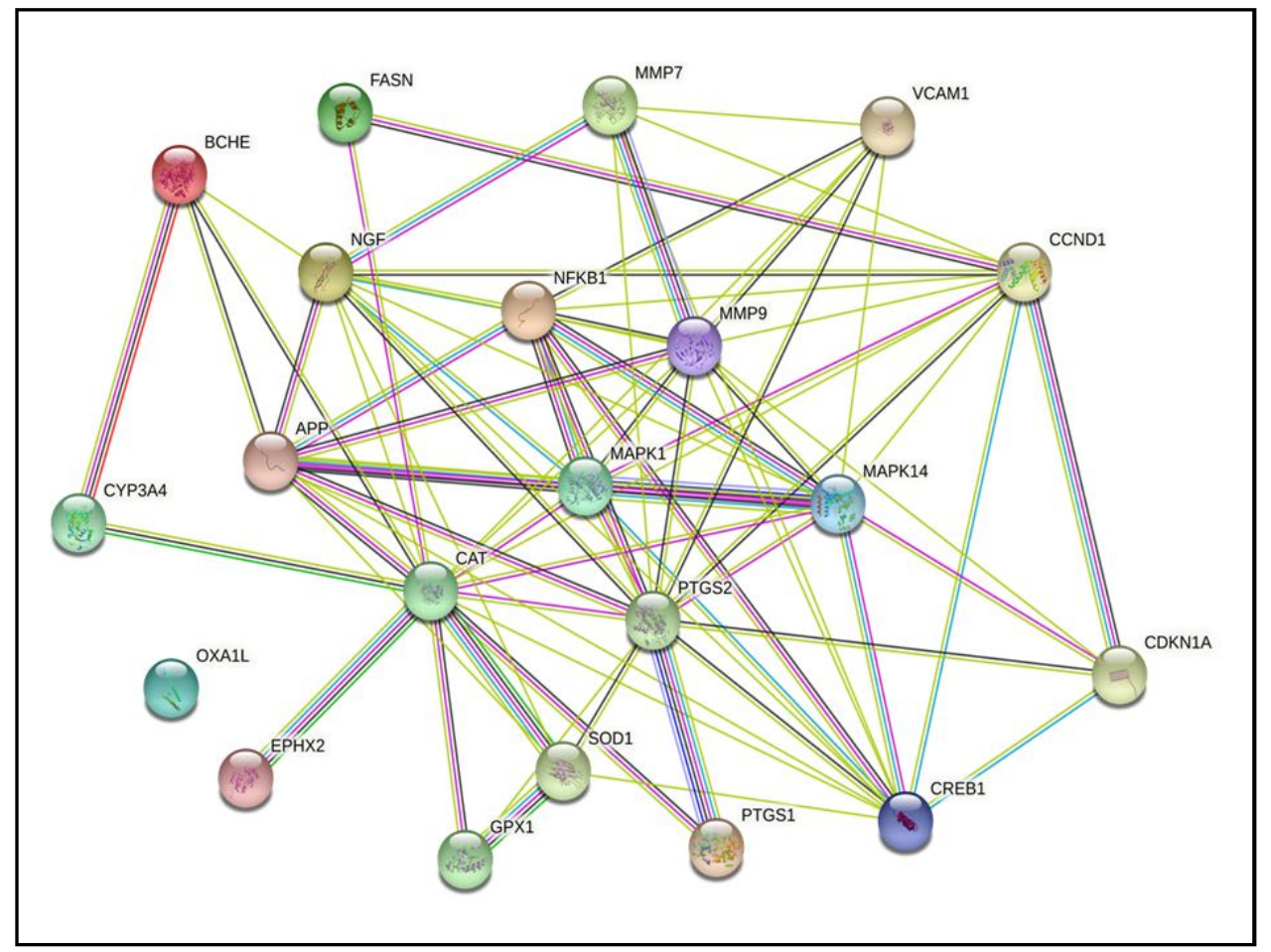

Figure 1: Protein-protein interaction of regulated proteins by oleuropein.

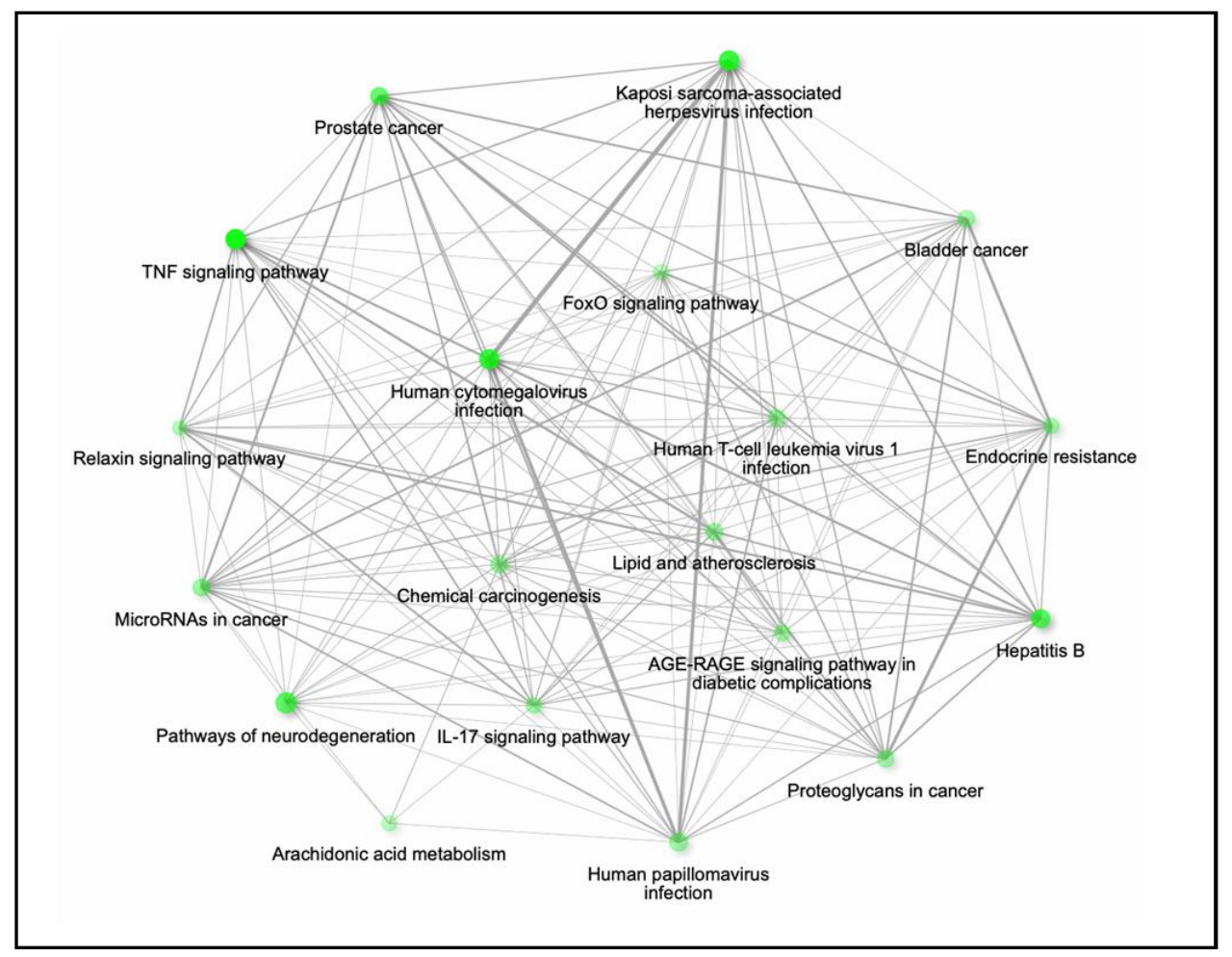

Figure 2: Top related pathways construction according to KEGG enrichment from STRING database. 
Table 4: KEGG enrichment analysis of modulated proteins

\begin{tabular}{|c|c|c|c|c|c|c|}
\hline \#term ID & Term description & $\begin{array}{l}\text { Observed } \\
\text { gene } \\
\text { count }\end{array}$ & $\begin{array}{l}\text { Back } \\
\text { ground } \\
\text { gene } \\
\text { count }\end{array}$ & Strength & $\begin{array}{l}\text { False } \\
\text { discovery } \\
\text { rate }\end{array}$ & $\begin{array}{l}\text { Matching proteins in the } \\
\text { network (labels) }\end{array}$ \\
\hline hsa04668 & TNF signaling pathway & 8 & 112 & 1.82 & $8.43 \mathrm{e}-09$ & $\begin{array}{l}\text { MAPK1, NFKB1, MAPK14, } \\
\text { VCAM1, PTGS2, MMP7, MMP9, } \\
\text { CREB1 }\end{array}$ \\
\hline hsa05167 & $\begin{array}{l}\text { Kaposi sarcoma-associated } \\
\text { herpesvirus infection }\end{array}$ & 8 & 194 & 1.56 & $1.32 \mathrm{e}-07$ & $\begin{array}{l}\text { CDKN1A, CREB1, MAPK14, } \\
\text { FAS, NFKB1, MAPK1, PTGS2, } \\
\text { CCND1 }\end{array}$ \\
\hline hsa05163 & Human cytomegalovirus infection & 8 & 224 & 1.5 & $1.86 \mathrm{e}-07$ & $\begin{array}{l}\text { MAPK1, NFKB1, CCND1, } \\
\text { MAPK14, PTGS2, CDKN1A, } \\
\text { CREB1, FAS }\end{array}$ \\
\hline hsa05161 & Hepatitis B & 7 & 161 & 1.57 & $1.04 \mathrm{e}-06$ & $\begin{array}{l}\text { CDKN1 A, CREB1, MAPK14, FAS, } \\
\text { MMP9, NFKB1, MAPK1 }\end{array}$ \\
\hline hsa05215 & Prostate cancer & 6 & 97 & 1.79 & $1.32 \mathrm{e}-07$ & $\begin{array}{l}\text { MAPK1, NFKB1, CCND1, MMP9, } \\
\text { CDKN1A, CREB1 }\end{array}$ \\
\hline hsa05206 & MicroRNAs in cancer & 6 & 161 & 1.56 & $1.04 \mathrm{e}-06$ & $\begin{array}{l}\text { MAPK1, NFKB1, CCND1, PTGS2, } \\
\text { MMP9, CDKN1A }\end{array}$ \\
\hline hsa04657 & IL-17 signaling pathway & 5 & 92 & 1.73 & $2.55 \mathrm{e}-06$ & $\begin{array}{l}\text { MAPK1, NFKB1, MAPK14, } \\
\text { PTGS2, MMP9 }\end{array}$ \\
\hline hsa01522 & Endocrine resistance & 5 & 95 & 1.71 & $2.60 \mathrm{e}-06$ & $\begin{array}{l}\text { MAPK1, CCND1, MAPK14, } \\
\text { MMP9, CDKN1A }\end{array}$ \\
\hline hsa04933 & $\begin{array}{l}\text { AGE-RAGE signaling pathway in } \\
\text { diabetic complications }\end{array}$ & 5 & 98 & 1.7 & $2.68 \mathrm{e}-06$ & $\begin{array}{l}\text { MAPK1, NFKB1, CCND1, } \\
\text { MAPK14, VCAM1 }\end{array}$ \\
\hline hsa05166 & $\begin{array}{l}\text { Human T-cell leukemia virus } 1 \\
\text { infection }\end{array}$ & 6 & 211 & 1.44 & $2.68 \mathrm{e}-06$ & $\begin{array}{l}\text { MAPK1, NFKB1, CCND1, MMP7, } \\
\text { CDKN1A, CREB1 }\end{array}$ \\
\hline hsa05219 & Bladder cancer & 4 & 41 & 1.98 & $4.73 e-06$ & $\begin{array}{l}\text { MAPK1, CCND1, MMP9, } \\
\text { CDKN1A }\end{array}$ \\
\hline hsa04068 & FoxO signaling pathway & 5 & 127 & 1.59 & $6.97 e-06$ & $\begin{array}{l}\text { MAPK1, CCND1, MAPK14, CAT, } \\
\text { CDKN1A }\end{array}$ \\
\hline hsa04926 & Relaxin signaling pathway & 5 & 128 & 1.58 & $6.97 e-06$ & $\begin{array}{l}\text { MAPK1, NFKB1, MAPK14, } \\
\text { MMP9, CREB1 }\end{array}$ \\
\hline hsa04218 & Cellular senescence & 5 & 150 & 1.51 & $1.33 e-05$ & $\begin{array}{l}\text { MAPK1, NFKB1, CCND1, } \\
\text { MAPK14, CDKN1A }\end{array}$ \\
\hline hsa05165 & Human papillomavirus infection & 6 & 325 & 1.26 & $2.14 \mathrm{e}-05$ & $\begin{array}{l}\text { MAPK1, NFKB1, CCND1, PTGS2, } \\
\text { CDKN1A, CREB1 }\end{array}$ \\
\hline hsa04917 & Prolactin signaling pathway & 4 & 69 & 1.75 & $2.33 \mathrm{e}-05$ & $\begin{array}{l}\text { MAPK1, NFKB1, CCND1, } \\
\text { MAPK14 }\end{array}$ \\
\hline hsa05140 & Leishmaniasis & 4 & 70 & 1.75 & $2.33 e-05$ & $\begin{array}{l}\text { MAPK1, NFKB1, MAPK14, } \\
\text { PTGS2 }\end{array}$ \\
\hline hsa04151 & PI3K-Akt signaling pathway & 6 & 350 & 1.22 & $2.57 \mathrm{e}-05$ & $\begin{array}{l}\text { MAPK1, NFKB1, CCND1, NGF, } \\
\text { CDKN1A, CREB1 }\end{array}$ \\
\hline hsa05203 & Viral carcinogenesis & 5 & 182 & 1.43 & $2.57 \mathrm{e}-05$ & $\begin{array}{l}\text { MAPK1, NFKB1, CCND1, } \\
\text { CDKN1A, CREB1 }\end{array}$ \\
\hline
\end{tabular}




\begin{tabular}{|c|c|c|c|c|c|c|}
\hline hsa05212 & Pancreatic cancer & 4 & 73 & 1.73 & $2.57 \mathrm{e}-05$ & MAPK1, NFKB1, CCND1, CDKN1A \\
\hline hsa05220 & Chronic myeloid leukemia & 4 & 75 & 1.72 & $2.57 \mathrm{e}-05$ & MAPK1, NFKB1, CCND1, CDKN1A \\
\hline hsa05205 & Proteoglycans in cancer & 5 & 196 & 1.4 & $3.06 \mathrm{e}-05$ & $\begin{array}{l}\text { MAPK1, CCND1, MAPK14, } \\
\text { MMP9, CDKN1A }\end{array}$ \\
\hline hsa05222 & Small cell lung cancer & 4 & 92 & 1.63 & $4.88 \mathrm{e}-05$ & NFKB1, CCND1,PTGS2,CDKN1A \\
\hline hsa04625 & C-type lectin receptor signaling pathway & 4 & 102 & 1.58 & $6.95 e-05$ & MAPK1, NFKB1, MAPK14, PTGS2 \\
\hline hsa04722 & Neurotrophin signaling pathway & 4 & 114 & 1.54 & 0.00010 & MAPK1,NFKB 1,MAPK14,NGF \\
\hline hsa04380 & Osteoclast differentiation & 4 & 122 & 1.51 & 0.00013 & MAPK1,NFKB 1,MAPK14,CREB 1 \\
\hline hsa05216 & Thyroid cancer & 3 & 36 & 1.91 & 0.00014 & MAPK1,CCND1,CDKN1A \\
\hline hsa05418 & Fluid shear stress and atherosclerosis & 4 & 130 & 1.48 & 0.00015 & NFKB 1,MAPK14,VCAM1,MMP9 \\
\hline hsa05200 & Pathways in cancer & 6 & 517 & 1.06 & 0.00016 & $\begin{array}{l}\text { MAPK1, NFKB1, CCND1, PTGS2, } \\
\text { MMP9, CDKN1A }\end{array}$ \\
\hline hsa04921 & Oxytocin signaling pathway & 4 & 149 & 1.42 & 0.00024 & MAPK1,CCND1,PTGS2,CDKN1A \\
\hline hsa04934 & Cushing syndrome & 4 & 153 & 1.41 & 0.00026 & MAPK1,CCND1,CDKN1A,CREB 1 \\
\hline hsa05160 & Hepatitis C & 4 & 156 & 1.4 & 0.00027 & MAPK1,NFKB1,CCND1,CDKN1A \\
\hline hsa05152 & Tuberculosis & 4 & 168 & 1.37 & 0.00034 & MAPK1,NFKB 1,MAPK14,CREB 1 \\
\hline hsa04370 & VEGF signaling pathway & 3 & 57 & 1.71 & 0.00040 & MAPK1,MAPK14,PTGS2 \\
\hline hsa05213 & Endometrial cancer & 3 & 57 & 1.71 & 0.00040 & MAPK1,CCND1,CDKN1A \\
\hline hsa00590 & Arachidonic acid metabolism & 3 & 61 & 1.68 & 0.00046 & PTGS2,GPX1,EPHX2 \\
\hline hsa05169 & Epstein-Barr virus infection & 4 & 193 & 1.31 & 0.00052 & $\begin{array}{l}\text { NFKB1, CCND1, MAPK14, } \\
\text { CDKN1A }\end{array}$ \\
\hline hsa05221 & Acute myeloid leukemia & 3 & 66 & 1.65 & 0.00055 & MAPK1,NFKB 1,CCND 1 \\
\hline hsa05223 & Non-small cell lung cancer & 3 & 68 & 1.64 & 0.00058 & MAPK1,CCND1,CDKN1 A \\
\hline hsa05214 & Glioma & 3 & 72 & 1.61 & 0.00067 & MAPK1,CCND1,CDKN1A \\
\hline hsa05218 & Melanoma & 3 & 72 & 1.61 & 0.00067 & MAPK1,CCND1,CDKN1A \\
\hline hsa05133 & Pertussis & 3 & 74 & 1.6 & 0.00069 & MAPK1, NFKB1, MAPK14 \\
\hline hsa04146 & Peroxisome & 3 & 79 & 1.57 & 0.00081 & CAT, SOD1, EPHX2 \\
\hline hsa05210 & Colorectal cancer & 3 & 82 & 1.55 & 0.00088 & MAPK1, CCND1, CDKN1A \\
\hline hsa04211 & Longevity regulating pathway & 3 & 87 & 1.53 & 0.0010 & NFKB1, CAT, CREB 1 \\
\hline hsa04658 & Th1 and Th2 cell differentiation & 3 & 87 & 1.53 & 0.0010 & MAPK1, NFKB1, MAPK14 \\
\hline hsa05235 & $\begin{array}{l}\text { PD-L1 expression and PD-1 checkpoint } \\
\text { pathway in cancer }\end{array}$ & 3 & 88 & 1.52 & 0.0010 & MAPK1, NFKB1, MAPK14 \\
\hline hsa05020 & Prion disease & 4 & 265 & 1.17 & 0.0013 & MAPK1, MAPK14, SOD1, CREB1 \\
\hline hsa04064 & NF-kappa B signaling pathway & 3 & 101 & 1.46 & 0.0014 & NFKB1, VCAM1, PTGS2 \\
\hline hsa04066 & HIF-1 signaling pathway & 3 & 106 & 1.44 & 0.0014 & MAPK1, NFKB1, CDKN1A \\
\hline hsa04620 & Toll-like receptor signaling pathway & 3 & 101 & 1.46 & 0.0014 & MAPK1, NFKB1, MAPK14 \\
\hline hsa04659 & Th17 cell differentiation & 3 & 101 & 1.46 & 0.0014 & MAPK1, NFKB1, MAPK14 \\
\hline hsa04660 & $\mathrm{T}$ cell receptor signaling pathway & 3 & 101 & 1.46 & 0.0014 & MAPK1, NFKB1, MAPK14 \\
\hline hsa04928 & $\begin{array}{l}\text { Parathyroid hormone synthesis, } \\
\text { secretion and action }\end{array}$ & 3 & 103 & 1.45 & 0.0014 & MAPK1, CDKN1A, CREB1 \\
\hline hsa05142 & Chagas disease & 3 & 99 & 1.47 & 0.0014 & MAPK1, NFKB1, MAPK14 \\
\hline hsa05145 & Toxoplasmosis & 3 & 105 & 1.45 & 0.0014 & MAPK1, NFKB1, MAPK14 \\
\hline hsa04010 & MAPK signaling pathway & 4 & 288 & 1.13 & 0.0015 & MAPK1, NFKB1, MAPK14, NGF \\
\hline
\end{tabular}




\begin{tabular}{|c|c|c|c|c|c|c|}
\hline hsa04670 & Leukocyte transendothelial migration & 3 & 109 & 1.43 & 0.0015 & MAPK14, VCAM1, MMP9 \\
\hline hsa04726 & Serotonergic synapse & 3 & 108 & 1.43 & 0.0015 & MAPK1, APP, PTGS2 \\
\hline hsa04071 & Sphingolipid signaling pathway & 3 & 116 & 1.4 & 0.0017 & MAPK1, NFKB1, MAPK14 \\
\hline hsa04935 & $\begin{array}{l}\text { Growth hormone synthesis, secretion } \\
\text { and action }\end{array}$ & 3 & 118 & 1.4 & 0.0018 & MAPK1, MAPK14, CREB1 \\
\hline hsa04152 & AMPK signaling pathway & 3 & 120 & 1.39 & 0.0019 & CCND1, FASN, CREB1 \\
\hline hsa05135 & Yersinia infection & 3 & 125 & 1.37 & 0.0021 & MAPK1, NFKB1, MAPK14 \\
\hline hsa04210 & Apoptosis & 3 & 132 & 1.35 & 0.0024 & MAPK1, NFKB1, NGF \\
\hline hsa04915 & Estrogen signaling pathway & 3 & 133 & 1.34 & 0.0024 & MAPK1, MMP9, CREB1 \\
\hline hsa05014 & Amyotrophic lateral sclerosis & 4 & 352 & 1.05 & 0.0028 & MAPK14, CAT, SOD1, GPX1 \\
\hline hsa04261 & Adrenergic signaling in cardiomyocytes & 3 & 147 & 1.3 & 0.0029 & MAPK1, MAPK14, CREB 1 \\
\hline hsa04723 & Retrograde endocannabinoid signaling & 3 & 145 & 1.31 & 0.0029 & MAPK1, MAPK14, PTGS2 \\
\hline hsa05010 & Alzheimer disease & 4 & 355 & 1.04 & 0.0029 & MAPK1, NFKB1, APP, PTGS2 \\
\hline hsa05224 & Breast cancer & 3 & 145 & 1.31 & 0.0029 & MAPK1, CCND1, CDKN1A \\
\hline hsa05226 & Gastric cancer & 3 & 144 & 1.31 & 0.0029 & MAPK1, CCND1, CDKN1A \\
\hline hsa05225 & Hepatocellular carcinoma & 3 & 160 & 1.26 & 0.0037 & MAPK1, CCND1, CDKN1A \\
\hline hsa05202 & Transcriptional misregulation in cancer & 3 & 171 & 1.23 & 0.0044 & NFKB 1, MMP9, CDKN1A \\
\hline hsa04621 & NOD-like receptor signaling pathway & 3 & 174 & 1.23 & 0.0045 & MAPK1, NFKB1, MAPK14 \\
\hline hsa05130 & Pathogenic Escherichia coli infection & 3 & 187 & 1.2 & 0.0055 & MAPK1, NFKB1, MAPK14 \\
\hline hsa04015 & Rap1 signaling pathway & 3 & 202 & 1.16 & 0.0067 & MAPK1, MAPK14, NGF \\
\hline hsa05170 & $\begin{array}{l}\text { Human immunodeficiency virus } 1 \\
\text { infection }\end{array}$ & 3 & 204 & 1.16 & 0.0068 & MAPK1, NFKB1, MAPK14 \\
\hline hsa04024 & cAMP signaling pathway & 3 & 208 & 1.15 & 0.0071 & MAPK1, NFKB1, CREB 1 \\
\hline hsa05030 & Cocaine addiction & 2 & 49 & 1.6 & 0.0071 & NFKB1, CREB 1 \\
\hline hsa05132 & Salmonella infection & 3 & 209 & 1.15 & 0.0071 & MAPK1, NFKB1, MAPK14 \\
\hline hsa05131 & Shigellosis & 3 & 218 & 1.13 & 0.0079 & MAPK1, NFKB1, MAPK14 \\
\hline hsa04014 & Ras signaling pathway & 3 & 226 & 1.11 & 0.0086 & MAPK1, NFKB1, NGF \\
\hline hsa04213 & $\begin{array}{l}\text { Longevity regulating pathway - multiple } \\
\text { species }\end{array}$ & 2 & 61 & 1.51 & 0.0102 & CAT, SOD1 \\
\hline hsa04664 & Fc epsilon RI signaling pathway & 2 & 66 & 1.47 & 0.0117 & MAPK1, MAPK14 \\
\hline hsa05211 & Renal cell carcinoma & 2 & 66 & 1.47 & 0.0117 & MAPK1, CDKN1A \\
\hline hsa05120 & $\begin{array}{l}\text { Epithelial cell signaling in Helicobacter } \\
\text { pylori infection }\end{array}$ & 2 & 67 & 1.47 & 0.0118 & NFKB1, MAPK14 \\
\hline hsa01524 & Platinum drug resistance & 2 & 70 & 1.45 & 0.0127 & MAPK1, CDKN1A \\
\hline hsa04622 & RIG-I-like receptor signaling pathway & 2 & 70 & 1.45 & 0.0127 & NFKB 1, MAPK14 \\
\hline hsa01100 & Metabolic pathways & 6 & 1447 & 0.61 & 0.0131 & $\begin{array}{l}\text { CAT, FASN, CYP3A4, PTGS2, } \\
\text { GPX1, EPHX2 }\end{array}$ \\
\hline hsa04115 & p53 signaling pathway & 2 & 72 & 1.43 & 0.0131 & CCND1, CDKN1A \\
\hline hsa04918 & Thyroid hormone synthesis & 2 & 74 & 1.42 & 0.0135 & CREB1, GPX1 \\
\hline hsa05204 & Chemical carcinogenesis & 2 & 75 & 1.42 & 0.0137 & CYP3A4, PTGS2 \\
\hline hsa04662 & B cell receptor signaling pathway & 2 & 78 & 1.4 & 0.0146 & MAPK1, NFKB1 \\
\hline hsa04012 & ErbB signaling pathway & 2 & 83 & 1.37 & 0.0162 & MAPK1, CDKN1A \\
\hline hsa05016 & Huntington disease & 3 & 298 & 0.99 & 0.0162 & SOD1, CREB1, GPX1 \\
\hline hsa04912 & GnRH signaling pathway & 2 & 89 & 1.34 & 0.0182 & MAPK1, MAPK14 \\
\hline
\end{tabular}




\begin{tabular}{|c|c|c|c|c|c|c|}
\hline hsa04713 & Circadian entrainment & 2 & 92 & 1.33 & 0.0192 & MAPK1, CREB1 \\
\hline hsa04750 & $\begin{array}{l}\text { Inflammatory mediator regulation } \\
\text { of TRP channels }\end{array}$ & 2 & 94 & 1.32 & 0.0198 & MAPK14, NGF \\
\hline hsa04914 & $\begin{array}{l}\text { Progesterone-mediated oocyte } \\
\text { maturation }\end{array}$ & 2 & 94 & 1.32 & 0.0198 & MAPK1, MAPK14 \\
\hline hsa04916 & Melanogenesis & 2 & 95 & 1.31 & 0.0198 & MAPK1, CREB1 \\
\hline hsa04931 & Insulin resistance & 2 & 107 & 1.26 & 0.0245 & NFKB 1, CREB 1 \\
\hline hsa04725 & Cholinergic synapse & 2 & 110 & 1.25 & 0.0256 & MAPK1, CREB1 \\
\hline hsa04919 & Thyroid hormone signaling pathway & 2 & 119 & 1.22 & 0.0295 & MAPK1, CCND1 \\
\hline hsa04110 & Cell cycle & 2 & 120 & 1.21 & 0.0297 & CCND1, CDKN1A \\
\hline hsa04114 & Oocyte meiosis & 2 & 120 & 1.21 & 0.0297 & MAPK1, MAPK14 \\
\hline hsa04611 & Platelet activation & 2 & 122 & 1.21 & 0.0300 & MAPK1, MAPK14 \\
\hline hsa04728 & Dopaminergic synapse & 2 & 128 & 1.18 & 0.0326 & MAPK14, CREB1 \\
\hline hsa04371 & Apelin signaling pathway & 2 & 131 & 1.17 & 0.0337 & MAPK1, CCND1 \\
\hline hsa04910 & Insulin signaling pathway & 2 & 133 & 1.17 & 0.0344 & MAPK1, FASN \\
\hline hsa05162 & Measles & 2 & 138 & 1.15 & 0.0366 & NFKB1, CCND1 \\
\hline hsa04550 & $\begin{array}{l}\text { Signaling pathways regulating } \\
\text { pluripotency of stem cells }\end{array}$ & 2 & 140 & 1.15 & 0.0372 & MAPK1, MAPK14 \\
\hline hsa05034 & Alcoholism & 2 & 144 & 1.13 & 0.0389 & MAPK1, CREB 1 \\
\hline hsa04310 & Wnt signaling pathway & 2 & 154 & 1.1 & 0.0438 & CCND1, MMP7 \\
\hline hsa04630 & JAK-STAT signaling pathway & 2 & 160 & 1.09 & 0.0467 & CCND1, CDKN1A \\
\hline hsa04022 & cGMP-PKG signaling pathway & 2 & 162 & 1.08 & 0.0473 & MAPK1, CREB1 \\
\hline hsa05164 & Influenza A & 2 & 165 & 1.07 & 0.0486 & MAPK1, NFKB 1 \\
\hline
\end{tabular}

\section{Discussion}

Network-based approaches is an emerging discipline to elucidate potential molecular mechanisms and pharmacological properties of natural compounds (Feng et al., 2017; Yang et al., 2020). Hence, target genes, proteins, and molecular pathways modulated by oleuropein were identified in this research, and twenty-one genes were determined as top interacting genes which are regulated by oleuropein in human genome. Oleuropein causes an increase in some of these genes, while it causes a decrease in some of them. For instance, oleuropein increases the activity of CAT genes, which encodes catalase, a key enzyme related to oxidative stress and immune system. Further, CAT protein co-treated with SOD1 protein inhibits the reaction of superoxide, resulting in increased response to oxidative stress and cell death. Therefore, oleuropein may behave as a powerful antioxidant, immune stimulator and cancer preventive agent (Shi et al., 2017; Modi et al., 2019; Guo et al., 2020). NGF is another important gene that encodes a secreted protein with nerve growth stimulating activity and its expression level is induced by oleuropein. The expression level of NGF, associated with neuropathy, hereditary sensory and corneal ulcer, is induced by oleuropein (De Nicoló et al., 2013; Carito et al., 2015).

On the contrary, oleuropein suppresses activity of MAPK1 and MAPK14 proteins, and also promotes the reaction of TNF protein which are results in increased apoptotic process, as well as decreased cell proliferation (Feng et al., 2017; Castejon et al., 2019). It can be clearly conducted that oleuropein play significant roles as a valuable anticancer agent and inflammatory mediator. Likewise, NFKB1, a pleiotropic transcription factor, is the endpoint of a series of signal transduction events such as inflammation, immunity, differentiation, cell growth, tumorigenesis and apoptosis (Aggarwal et al., 2021). In addition, oleuropein reduces the levels of FASN genes, encoding a multifunctional protein involved in the anabolic conversion of dietary carbohydrates to fat in mammals. Increased expression of this protein is found to be associated with fatty liver disease and non-alcoholic fatty liver disease, which supports the hypocholesterolemic effect of oleuropein as a cholesterol antagonist (Priore et al., 2014; Hadrich et al., 2016). Besides hypocholesterolemic effect, oleuropein have been shown to induce anticancer effects by suppressing FASN protein expression (Menendez et al., 2008; Notarnicola et al., 2011). The expression of CREB1 is significantly reduced by oleuropein, that contribute to decrease the expressions of genes associated with thermogenesis, mitochondrial biogenesis, and oxidative phosphorylation. Inhibition of CREB activity resulted in inhibition of cell proliferation (Corona et al., 2007; Gao et al., 2020).

Similarly, expression level of MMP-7 and MMP-9 are downregulated by oleuropein that resulted in increased cellular response to reactive oxygen species, and decreased in cell migration. These MMP proteins are involved in the breakdown of extracellular matrix in normal physiological processes, such as embryonic development, reproduction, and tissue remodeling, as well as in disease processes, such as arthritis and metastasis (Feng et al., 2017; Sherif and AlGayyar, 2018). VCAM1 is another protein that the expression levels are downregulated by oleuropein, which inhibits the reaction of 
lipopolysaccharides, tetradecanoylphorbol acetate, and tumor necrosis factor $\alpha$ (TNF- $\alpha$ ) protein results in increased expression of VCAM1 protein. Oleuropein suppresses the endothelial adhesion molecule expression, thus revealing the notable antioxidant, anticancer and anti-inflammatory capacities of oleuropein (Turner et al., 2005; Dell'Agli et al., 2006).

Based on the PPI network analyses, MAPK14, CREB1, NFKB1, NGF, MMP9, APP, and PTGS2 are determined as the core proteins. Among them, MAPK14 (mitogen-activated protein kinase), also known as CSBP, CSBP1, CSBP2, CSPB1, EXIP, Mxi2, PRKM14, PRKM15, RK, SAPK2A, p38, p38ALPHA, is serin-threonine kinase protein, which is a significant component of the MAP kinase signal transduction pathway evoked by extracellular stimuli such as proinflammatory cytokines or physical stress leading to direct activation of transcription factors. CREB1 is a basic leucine zipper protein involved in different cellular processes including the synchronization of circadian rhythmicity and the differentiation of adipose cells. NFKB1 and NGF play roles as transcription factor, of which NFKB1 is involved in NF-kappa-B signaling pathway and stimulus related to many biological processes including inflammation, immunity, differentiation, cell growth, tumorigenesis and apoptosis, whilst NGF is involved in nerves system and act important roles for the development and maintenance of the sympathetic and sensory nervous systems. PTGS2, also named as cyclooxygenase-2, converts arachidonate to prostaglandin $\mathrm{H} 2$ that is responsible for production of inflammatory prostaglandins, which is closely related to increased cell adhesion, phenotypic changes, resistance to apoptosis and tumor angiogenesis in cancer. Finally, MMP9, a metalloproteinase protein located in extracellular matrix, is associated with pathways in inflammation, cancer and neurodegeneration (Sherif and Al-Gayyar, 2018; Cuyàs et al., 2019; Ganeshpurkar et al., 2019; Zhang et al., 2019; Gao et al., 2020).

In addition, target pathways modulated by oleuropein were identified in the presented research. It is well-acknowledged that multiple signaling pathways interact with each other in the metabolic processes normally occurred in living organisms. According to the KEGG pathway enrichment, most of the genes modulated by oleuropein are closely related to oxidative stress, immune system and neurodegeneration, as well as cancer. Among the top oleuropeinregulated pathways, MAPK1, NFKB1, MAPK14, VCAM1, PTGS2, MMP7, MMP9, CREB1 genes are involved in TNF signaling pathway, which are related to regulate wide range of intracellular signal pathways including apoptos is and cell survival as well as inflammation and immunity. The genes of MAPK1, NFKB1, CCND1, CDKN1A, CREB1, MAPK14, VCAM1, PTGS2, MMP7, and MMP9, whose expressions are regulated by oleuropein, are involved in cancerrelated signaling pathways including, apoptosis, cell cycle, cell death, cellular proliferation, and metastasis. Consistent with the findings from this bioinformatics-based study, previous reports show that dietary oleuropein suppresses cancer-related pathways such as Janus kinase/signal transducer and activator of transcription (JAK/STAT), nuclear transcription factor-kappa B (NF- $\beta B$ ), mitogen-activated protein kinases (MAPKs), and inflammasome nucleotide-binding domain, leucine-rich repeats-containing family, pyrin domaincontaining-3 (NLRP3) signaling pathways (Ratan et al., 2017; Castejon et al., 2019; Hsu et al., 2021; Mounika and Hymavathi, 2021). On the other hand, oleuropein has also participated in fatty acid synthesis pathways that demonstrated by previous studies
(Priore et al., 2014; Hadrich et al., 2016). As revealed in previous studies, oleuropein has been demonstrated to be an important pharmaceutical resource for drug targets, in agreement with these findings from network-based molecular and pharmacological analyzes.

\section{Conclusion}

Oleuropein is a promising phenolic compound that predominantly obtained from O.europaea (olive tree), and proven to have significant biological activities in the human body. TNF signaling pathway, micro RNAs in cancer, IL-17 signaling pathway, Kaposi sarcoma-associated herpesvirus infection, human cytomegalovirus infection, hepatitis $\mathrm{B}$, prostate cancer pathway, endocrine resistance, proteoglycans in cancer, and neurodegenerative signaling pathways involved in cell proliferation, oxidative stress, metastasis, apoptosis, neurodegeneration, fatty acid synthesis, and catabolic processes were defined as the top pathways regulated by oleuropein. Taken together, MAPK1, NFKB1, MAPK14, VCAM1, NGF, PTGS2, MMP7, MMP9, and CREB1 are main core proteins involved in top the signaling pathways, and these proteins and targets may be the key points of the therapeutic potentials of oleuropein. Based on our network pharmacological analysis, oleuropein may exert a wide range of pharmacological effect via multiple targets, pathways, and biological processes, thereby regulating the metabolism. Further studies are required to verify the clinical efficacy of oleuropein and its mechanisms of action.

\section{Conflict of interest}

The authors declares no conflicts of interest relevant to this article.

\section{References}

Aggarwal, V.; Kumar, G.; Aggarwal, D.; Yerer, M. B.; Cumaoglu,A.; Kumar, M. and Sethi, G. (2021). Cancer preventive role of olives and olive oil viamodu lation of apoptosis and nuclearfactor-kappa B activation. In Olives and Olive Oil in Health and Disease Prevention, pp:377-388. Academic Press.

Aoki-Kinoshita, K.F. and Kanehisa, M. (2007). Gene annotation and pathway mapping in KEGG. In Comparative Genomics, pp:71-91. Human Press.

Athanasios, A.; Charalampos, V. and Vasileios, T. (2017). Protein-protein interaction (PPI) network: Recent advances in drug discovery. Curr. Drug Metab., 18(1):5-10.

Carito, V.; Ceccanti, M.; Chaldakov, G.; Tarani, L.; De Nicolò, S.; Ciafrè, S. and Fiore, M. (2015). Polyphenols, nerve growth factor, brain-derived neuro trophic factor, and the brain. In Bioactive Nutraceuticals and Dietary Supplements in Neurological and Brain Disease, pp: 65-71. Academic Press.

Castejon, M.L.; Sánchez-Hidalgo, M.; Aparicio-Soto, M.; Montoya, T.; MartínLaCave, I.; Fernández-Bolaños, J.G. and Alarcón-de-la-Lastra, C. (2019). Dietaryoleuropein and itsnewacyl-deriv ateattenuate murinelupus nephritis through HO-1/Nrf2 activation and suppressing JAK/STAT, $\mathrm{NF}-\kappa \mathrm{B}$, MAPK and NLRP3 inflamma some signaling pathways. J. Nutr. Biochem., 74:108-229.

Corona, G.; Deiana, M.; Incani,A.; Vauzour, D.; Dessì, M.A. and Spencer, J.P. (2007). Inhibition of $\mathrm{p} 38 / \mathrm{CREB}$ phosphorylation and COX-2 expression by olive oil polyphenols underlies their anti-proliferative effects. Biochem. Biophys. Res. Commun., 362(3):606-611.

Cuyàs, E.; Castillo, D.; Llorach-Parés, L.; Lozano-Sánchez, J.; Verdura, S.; NonellCanals, A. and Menendez, J. A. (2019). Computational de-orphanization of the olive oil biophenololeacein: Discovery of new metabolic and epigenetic targets. Food Chem.Toxicol., 131:110-529. 
Das, K. and Gezici, S. (2018). Secondary plant metabolites, their separation and identification, and role in human disease prevention. Ann. Phytomed., 7:13-24.

De Nicoló, S.; Tarani, L.; Ceccanti, M.; Maldini, M.; Natella, F.; Vania, A. and Fiore, M. (2013). Effects of olive polyphenols administration on nerve growth factor and brain-derived neurotrophic factor in the mousebrain. Nutr., 29(4):681-687.

Dell'Agli, M.; Fagnani, R.; Mitro, N.; Scurati, S.; Masciadri, M.; Mussoni, L.; and Caruso, D. (2006). Minor components of olive oil modulate proatherogenic adhesion molecules involved in endo the lial activation. J. Agric. Food Chem., 54(9):3259-3264.

Feng, Z.; Li, X.; Lin, J.; Zheng, W.; Hu, Z.; Xuan, J. and Pan, X. (2017). Oleuropein inhibits the IL-1 $\beta$-induced expression of inflammatory mediators by suppressing the activation of NF- $\kappa \mathrm{B}$ and MAPKs in human osteoarthritis chondrocytes. Food Func., 8(10):3737-3744.

Fishilevich, S.; Zimmerman, S.; Kohn, A.; Iny Stein, T.; Olender, T.; Kolker, E. and Lancet, D. (2016). Genic insights from integrated human proteomics in Gene Cards Database, pp:20-36.

Ganeshpurkar, A.; Swetha, R.; Kumar, D.; Gangaram, G.P.; Singh, R.; Gutti, G.; and Singh, S.K. (2019). Protein-protein interactions and aggregation inhibitors in Alzheimer's disease. Curr. Top. Med. Chem., 19(7): 501-533.

Gao, Y.; Li, X.; Xu, R.; Guo, Y.; Yin, H.; Tan, R. and Ya, B. (2020). Oleuropein improved post cerebral stroke cognitive function by promoting histone acetylation and phosphorylation of cAMP response element-binding protein in MCAO rats. Dose Response, 18(3): 1559325820950102

Guo, Q.; Li, F.; Duan, Y.; Wen, C.; Wang, W.; Zhang, L. and Yin, Y. (2020). Oxidative stress, nutritional antioxidants and beyond. Science China Life Sci., 63(6): $866-874$

Hadrich, F.; Mahmoudi, A.; Bouallagui, Z.; Feki, I.; Isoda, H.; Feve, B. and Sayadi, S. (2016). Evaluation of hypocholesterolemic effect of oleuropein in cholesterol-fed rats. Chem. Biol. Interact., 252:54-60.

Harel, A.; Inger, A.; Stelzer, G.; Strichman-Almashanu, L.; Dalah, I.; Safran, M. and Lancet, D. (2009). GIFtS: Annotation landscape analysis with Gene Cards BMC Bioinform., 10(1):1-11.

Hastings, J.; Owen, G.; Dekker, A.; Ennis, M.; Kale, N.; Muthukrishnan, V. and Steinbeck, C. (2016). ChEBI in 2016: Improved services and an expanding collection of metabolites. Nucleic Acids Res., 44(D1), D1214-D1219.

Hsu, M.L.; Huang, W.C.; Zhou, Y.R.; Hu, S.; Huang, C.H. and Wu, S.J. (2021) Oleuropein protects human retinal pigment epithelium cells from IL- $1 \beta$-induced inflammation by blocking MAPK/NF- $\kappa \mathrm{B}$ signaling pathways. Inflammation, pp:1-11.

Kanehisa, M.; Furumichi, M.; Tanabe, M.; Sato, Y. and Morishima, K. (2017). KEGG: New perspectives on genomes, pathways, diseases and drugs. Nucleic Acids Res., 45(D1):D353-D361.

Lagunin, A.; Ivanov, S.; Rudik, A.; Filimonov, D. and Poroikov, V. (2013). DIGEPPred: Web service for in silico prediction of drug-induced gene expression profiles based on structural formula. Bioinformatics, 29(16):2062e3. https://doi.org/10.1093/bioinformatics/btt322.
Menendez, J.A; Vazquez-Martin, A.; Oliveras-Ferraros, C.; Garcia-Villalba, R.; Carrasco-Pancorbo, A.; Fernandez-Gutierrez, A. and Segura-Carretero, A. (2008). Analyzing effects of extra-virgin olive oil polyphenols on breast cancer-associated fatty acid synthase protein expression using reverse-phase protein microarrays. Int. J. Mol. Med., 22(4):433-439.

Modi, F. D.; Bhavsar, S.K.; Patel, J.H.; Varia, R.D.; Modi, L.C.; Modi, M. and Kale, N. (2019). Pharmacokinetic profile of rutin after intramuscular administration in rats favoursits in vivo anti-inflammatory activity in carrageenan-induced rodent model of inflammation. Ann. Phytomedicine, 8(1):185-192.

Mounika, M. and Hymavathi, T.V. (2021). Nutrient and phytonutrient quality of nutricereals incorporated flour mix suitable for diabetics. Ann. Phytomedicine, 10(1):132-140.

Notarnicola, M.; Pisanti, S.; Tutino, V.; Bocale, D.; Rotelli, M.T.; Gentile, A. and Caruso, M.G. (2011). Effects of olive oil polyphenols on fatty acid synthase gene expression and activity in human colorectal cancer cells. Genes Nutr., 6(1):63-69.

Priore, P.; Siculella, L. and Gnoni, G.V. (2014). Extra virgin olive oil phenols down-regulate lipid synthesis in primary-cultured rat-hepatocytes. J. Nutr. Biochem., 25(7):683-691.

Ratan, V.; Dixit, S.; Srivastava, M.; Trivedi, S.; Mishra, A.; Srivastava, Y.K. and Srivastava, D.K. (2017). Computational structure prediction and analyze active ligand binding site of defense and lytic enzymes of Trichoderma harzianum. Ann. Phytomed., 7:143-160.

Sekeroglu N.; Erdogan, I. and Gezici, S. (2020). Kilis yaglik virgin olive oil: A novel source for omega-7 fatty acids, especially paullinic acid. The Sixth International Mediterranean Symposium on Medicinal and Aromatic Plants: MESMAP-6, October 15-17, 2020, Izmir, Turkey. pp:50 (Oral Presentation).www.mesmap.com

Sherif, I.O. and Al-Gayyar, M.M. (2018). Oleurope in potentiates anti-tumor activity of cisplatin against HepG2 through affecting pro NGF/NGF balance. Life Sci., 198:87-93.

Shi, C.; Chen, X.; Liu, Z; Meng, R.; Zhao, X.; Liu, Z and Guo, N. (2017). Oleuropein protects $\mathrm{L}-02$ cells against $\mathrm{H}_{2} \mathrm{O}_{2}$-induced oxidative stress by increasing SOD1, GPx1 and CAT expression. Biomed. Pharmacother., 85:740-748

Turner, R.; Etienne, N.; GarciaAlonso, M.; De Pascual-Teresa, S.; Minihane, A.M.; Weinberg, P.D. and Rimbach, G. (2005).Antioxidant and anti-atherogenic activities of olive oil phenolics. Int. J. Vitam. Nutr. Res., 75(1):6170 .

Wu, J.;Vallenius, T.;Ovaska, K.; Westermarck, J.;Mäkelä, T. P. and Hautaniemi, S. (2009). Integrated network analysis platform for protein-protein interac tions. Nat. Methods, 6(1):75-77.

Yang, L.;Li, H.;Yang, M.;Zhang, W.;Li, M.;Xu, Y. and Guo, S. (2020). Exploration in the mechanism of kaempferol for the treatment of gastric cancer based on network pharmacology. Bio. Med. Res. Int., pp:11-20.

Zhang, X.;Yang, P.;Luo, X.; Su, C.;Chen, Y.; Zhao, L. and Chen, Y. (2019). High olive oil diets enhance cervical tumour growth in mice: Transcrip to mean alysis for potentialc and idategenes and a path ways. Lipids Health Dis., 18(1):1-13. 